\title{
Incommensurate and Commensurate Structural Modulation in Martensitic Phases of FSMA
}

\author{
Lara Righi $^{1, a}$, Franca Albertini ${ }^{2}$, Antonio Paoluzi $^{2}$, Simone Fabbrici ${ }^{2}$, \\ Elena Villa ${ }^{3}$, Gianluca Calestani ${ }^{1}$, Stefano Besseghini ${ }^{3}$ \\ ${ }^{1}$ Dipartimento di Chimica Generale ed Inorganica, Chimica Analitica, Chimica Fisica, Università \\ di Parma, Viale G. Usberti 17/A, I-43100 Parma, Italy \\ ${ }^{2}$ IMEM, Consiglio Nazionale delle Ricerche, Parco Area delle Scienze 37/A, I-43010 Parma, Italy \\ ${ }^{3}$ IENI, Consiglio Nazionale delle Ricerche, Corso Promessi Sposi, 23900 Lecco, Italy \\ alara.righi@unipr.it
}

\begin{abstract}
Keywords: Ferromagnetic shape memory alloys; martensitic structure; incommensurate
\end{abstract} modulation; powder diffraction.

\begin{abstract}
Magnetic and structural properties in multifunctional FSMA (Ferromagnetic Shape Memory Alloys) belonging to Heusler family are frequently related to the occurrence of structural modulation in martensitic phases. The highest MFIS (Magnetic Field Induced Strain) effect has been observed in Ni-Mn-Ga alloys showing martensitic modulated structures. Depending on the composition, pressure and temperature conditions, this periodic structural distortion, consisting of shuffling of atomic layers along specific crystallographic directions, accompanies the martensitic transformation.

Over the years, different modulated martensitic structures have been observed and classified depending upon the periodicity of corresponding superstructure (nM with $n=3,5,6,7,12$ etc). On the other hand, it has been demonstrated that in most cases such structural modulation is incommensurate and the crystal structure can be solved by applying superspace approach. The crystallographic representation of different modulated structures, obtained by structure refinement on powder diffraction data, suggests a unified description where every different "nM" periodicity can be straightforwardly represented. It will be presented an overview illustrating structural features of several displacive modulated martensitic lattices. For a specific Ni-Mn-Ga composition, the evolution of structural modulation upon temperature change will be illustrated.
\end{abstract}

\section{Introduction}

Among ferromagnetic shape memory materials, Heusler alloys with composition Ni-Mn-Ga represent the most interesting and promising system for technological applications [1,2]. Fundamental properties at the basis of the huge MFIS are the ferromagnetic state with a Curie temperature $\left(\mathrm{T}_{\mathrm{C}}\right)$ exceeding $300 \mathrm{~K}$, the thermoelastic martensitic transformation (MT) generally below $\mathrm{T}_{\mathrm{C}}$, and the presence of a high magnetocrystalline anisotropy in the martensitic phase [3,4]. The MT temperature as well as crystal structure of the martensitic phase are controlled by Ni-Mn$\mathrm{Ga}$ compositional variations with respect to the stoichiometric $\mathrm{Ni}_{2} \mathrm{MnGa}$ [5]. Martensitic transformation consists of a lattice distortion (the so-called Bain deformation) of the cubic L2 ${ }_{1}$ type structure of parent phase [1]. As it is displayed in Fig.1, the symmetry of the product phase is properly described with a I-centred unit cell having the fundamental axes coinciding with the $[10 \overline{1}]$, [101] and [010] directions of the L $2{ }_{1}$ superstructure. However several diffraction experiments [6-9] proved the presence of additional weak reflections indicating that, in some cases, the martensitic structures are characterized by structural modulation. Such a structural distortion is determined by the instability of parent cubic structure which induces, besides the shear deformation of the lattice, shuffling of atomic layers along selected crystallographic directions. 


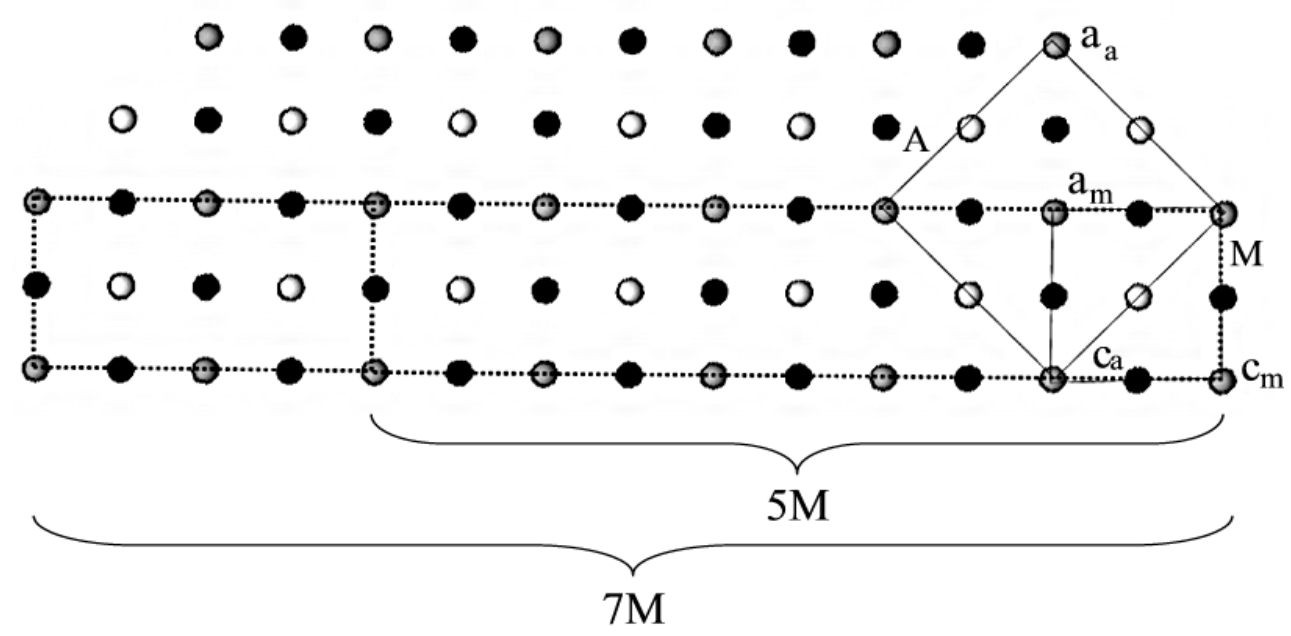

Fig. 1: Lattice relationships between cubic $\mathrm{L} 2_{1}$ superstructure $(A)$ and distorted martensitic lattice $(\mathrm{M})$; the typical commensurate modulated lattices $5 \mathrm{M}$ and $7 \mathrm{M}$ are also shown.

This structural feature has been initially detected by Webster et al. [6] in a $\mathrm{Ni}_{2} \mathrm{MnGa}$ single crystal studied at low temperature. The diffraction pattern is interpreted as derived by a five-fold superstructure $(5 \mathrm{M})$ involving five consecutive unit cells along the $[1 \overline{1} 0]$ direction of the $\mathrm{L} 2_{1}$ lattice. Afterwards recent investigations on Ni-Mn-Ga alloys have described a variety of different modulated structures [9]. The number of observed satellites (s) between the main reflections, is directly associated to superstructures constituted by 5, 6, 7, 10 or even 12 unit cells and commonly indicated as $5 \mathrm{M}, 6 \mathrm{M}, 7 \mathrm{M}, 10 \mathrm{M}$ and $12 \mathrm{M}$ [8-10], where $n \mathrm{M}$ is referred to the relationship $N=\mathrm{s}+1$. This classification excludes the occurrence of incommensurate modulation. Since the largest magnetostrain effects are observed for modulated martensites [11,12], the determination of their crystal structures assumes a crucial importance. In spite of the intense research dealing with the study of the magnetic and structural properties of the Ni-Mn-Ga system, the structural characterization of such phases is still a controversial matter. Therefore, we have investigated modulated martensitic phases by powder X-ray/neutron diffraction. Our results indicate the presence of incommensurate and commensurate modulation properly described within superspace approach [13-15]. The obtained crystal structures clarify a series of critical points concerning the structural study of Ni-Mn-Ga martensitic phases.

\section{The superspace approach}

This section provides the basic elements of superspace approach necessary to correctly interpret the structural results given in the next sections. A more comprehensive description of this theory can be found in ref.[16]. The diffraction pattern of incommensurate crystals are characterized by Bragg reflections that, even if sharp and well separated, do not belong to a lattice and cannot be indexed by three-integer indices. The analysis of the diffraction pattern reveals the presence of two distinct set of reflections. The first group is composed by intense peaks (main reflections) defining a conventional unit cell defined by $\mathbf{a}^{*}, \mathbf{b}^{*}$ and $\mathbf{c}^{*}$. The second set of reflections (satellites), usually weaker, is not indexed on the basis of the former group but it needs additional vectors. The indexing of the whole diffraction pattern requires the introduction of supplementary vectors and, as a result, the generic diffraction vector $\mathbf{H}$ has the form (in the case of mono-dimensional modulation):

$$
\mathbf{H}=\mathrm{h} \mathbf{a}^{*+\mathrm{k}} \mathbf{b}^{*+1} \mathrm{c}^{*}+\mathrm{mq}
$$

where the $\mathbf{q}$ vector is usually written as: 


$$
\mathbf{q}=\alpha \mathbf{a}^{*+}+\beta \mathbf{b}^{*+}+\gamma \mathbf{c} *
$$

Therefore each Bragg reflection has four indexes $h \mathrm{klm}$ corresponding to the three basic vector $\mathbf{a}^{*}$, $\mathbf{b}^{*}, \mathbf{c}^{*}$ and to the modulation vector $\mathbf{q}$. Depending on $\alpha, \beta$ and $\gamma$ coefficients it is possible to individuate two main classes of modulated structures. These coefficients are defined as the ratio between the modulation vector and the fundamental reciprocal vectors of the basic structure. If the value of this ratio is a rational number then the modulation is defined commensurate, otherwise, an irrational number determines an incommensurate modulation. The commensurate modulation (C) can be always expressed in terms of a three-dimensional superstructure. On the contrary, the crystallographic description of an incommensurate (IC) modulated crystal requires the introduction of the superspace.

In a conventional crystal, the atomic site $\bar{x}_{\mathrm{i}}$ (expressed by $x, y$ and $z$ fractional coordinates) is defined as:

$$
\bar{x}_{\mathrm{i}}=\mathrm{L}+\mathrm{x}_{\mathrm{i}}^{0}
$$

The L letter regards the primitive translation proper of the crystalline symmetry whereas $\mathrm{x}_{\mathrm{i}}{ }^{0}$ defines the atomic position of the $\mathrm{i}$-th atom in the unit cell. The structural modulation is derived by a periodic deviation involving atomic positions which are referred to the basic lattice defined by a, b and c principal vectors. In the incommensurate modulated crystal the generic position is converted in [6]:

$$
x_{i}=\bar{x}_{i}+u_{i}\left(\bar{x}_{4}\right)
$$

The superspace is an higher dimensional space where the three-dimensional physical space play the principal role with respect the additional dimensions: for this reason the incommensurate crystal is correlated to a $(3+1)$-dimensional space. The superspace enables to express all the periodicities present in the structure, included that related to the modulation showing a periodic length not commensurate to the three-dimensional basic lattice. The additional factor describes the displacive effect on the basic atomic positions. The function $u_{i}\left(\overline{\mathrm{x}}_{\mathrm{s} 4}\right)$ depends on the superspace coordinate $x_{4}$ and it is periodic with respect to the translation along the fourth axis. Accordingly to incommensurateness of the modulated structure the factor $u_{i}\left(\bar{x}_{4}\right)$ added to the un-distorted position of a specific atom is different for each adjacent unit cell destroying the three-dimensional translational symmetry of the crystal. Depending on the type of structural modulation the function $u\left(\bar{x}_{4}\right)$ can assume different forms. Nevertheless the modulation function is generally expanded into a Fourier series:

$$
u\left(\bar{x}_{4}\right)=\sum_{n=1}^{\infty} A_{n} \sin \left(2 \pi n \bar{x}_{4}\right)+B_{n} \cos \left(2 \pi n \bar{x}_{4}\right)
$$

The amplitudes $A_{n}$ and $B_{n}$ are the unknown parameters to be determined in order to characterize the structural modulation. The $n$ order of the Fourier series depends on the experimental data and it is correlated to the $m$ index of Eq.(1). The general atomic position $x_{i}\left(\bar{x}_{\mathrm{i}}, \bar{x}_{4}\right)$ depends from a couple of parameters, one derived from the average basic structure (external space), the second referred to the additional dimension of the superspace (internal space). The superspace permits a crystallographic description of the modulated crystal and consequently the whole symmetry of the system is expressed by the superspace groups. The superspace group symbol is composed by three parts:

(1) the space group of the basic structure; 
(2) the components of the q vector allowed by symmetry;

(3) the internal translational components along the fourth additional dimension corresponding to every symmetry operator in the fundamental space group.

\section{The "5M" family}

The thermally induced martensitic structure of stoichiometric $\mathrm{Ni}_{2} \mathrm{MnGa}$ has been usually interpreted as $5 \mathrm{M}$ modulated $[7,8]$. This was suggested by the observation, reported in the first studies concerning this Heusler alloy, of 4 satellites in single crystal diffraction pattern. Then, J.P. Brown and co-authors, on the basis of a Rietveld refinement with a orthorhombic seven-fold superstructure, indicated the same phase as belonging to the 7M family of modulated martensites [17]. The two descriptions seem to be in contrast and this discrepancy was never clarified. This structural problem has been therefore re-investigated taking into account all the suitable information present in literature [13]. It has been demonstrated that, in agreement with what observed by A. Zheludev et al. [18], this phase presents an incommensurate modulation whose structure has been solved within superspace approach [16]. The powder X-ray diffraction pattern collected at $100 \mathrm{~K}$ on a polycrystalline specimen of $\mathrm{Ni}_{2} \mathrm{MnGa}$ has been carefully analyzed. Besides the orthorhombic lattice

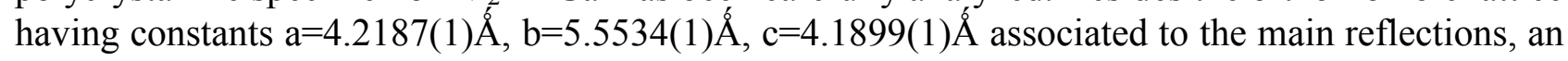
additional vector $\mathbf{q}=0.4248(2) \mathbf{c}^{*}$ has been introduced to index the observed satellites. The superspace group of the (3+1)-dimensional model is $\operatorname{Immm}(00 \gamma) \mathrm{s} 00$. The displacive modulation, involving the $(001)_{\mathrm{M}}$ atomic almost close-packed layers, is found to have a wave-like shape expressed by the first order of Eq. 6 (see Table1).
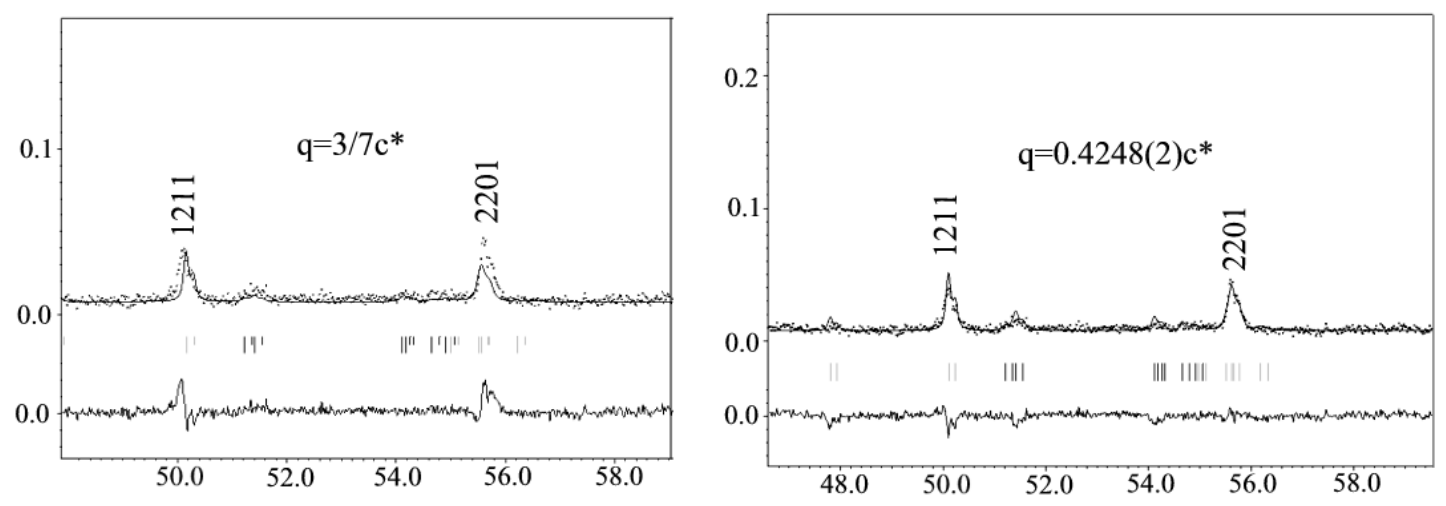

Fig. 2: Fragment of Rietveld plot obtained from powder X-ray diffraction data calculated with $\mathbf{q}$ fixed to $3 / 7 \mathbf{c}^{*}$ (left) and $\mathbf{q}$ refined (right).

It is interesting to note that $\mathbf{q}$ is $\sim 3 / 7 \mathbf{c}^{*}\left(=0.428 \mathbf{c}^{*}\right)$. This metrical coincidence induced P.J. Brown and co-workers to interpret the structure of this phase as $7 \mathrm{M}$ modulated [17]. Following this assumption it is possible to derive, from the $(3+1)$-dimensional, a seven-fold 3D model consisting of seven unit cells along the fundamental $c$ axis. The seven-fold superstructure must be viewed as an approximation very similar to the real incommensurate structure. Fig. 2 shows the same region of the plot of Rietveld refinements executed with $\mathbf{q}$ refined and fixed to $3 / 7 \mathbf{c}^{*}$. For the second case, owing to the incommensurate nature of the structure, the calculated satellites, even after refinement of the unit cell parameters, are slightly misaligned from the observed $2 \theta$ positions. The orthorhombic superstructure proposed by P.J. Brown contains several particularly short Ni-Mn and Ni-Ga distances (2.09 $\AA$ and $2.06 \AA$ respectively). These interatomic distances are unrealistic if compared to the characteristic bond lengths of these atomic species in intermetallic compounds. In Fig. 3 the two models are compared. The Brown's structure shows two wave-like modulations in anti-phase each other which lead to the shorter atomic interactions. The seven-fold superstructure calculated from superspace corresponds to a diffraction pattern composed by fundamental peaks 
spaced by 4 satellites in reciprocal space. This indicates that $\mathrm{Ni}_{2} \mathrm{MnGa}$ modulated structure possesses a " $5 \mathrm{M}$ " character; the modulation vector $\mathbf{q}$ slightly differs from commensurate period of $2 / 5 \mathbf{c}^{*}$, indeed, the product phase can be regarded as an nearly $5 M$ modulated having $\mathbf{q}=2 / 5(1+\delta) \mathbf{c}^{*}$ where $\delta$ regards the incommensurateness degree of the structure.

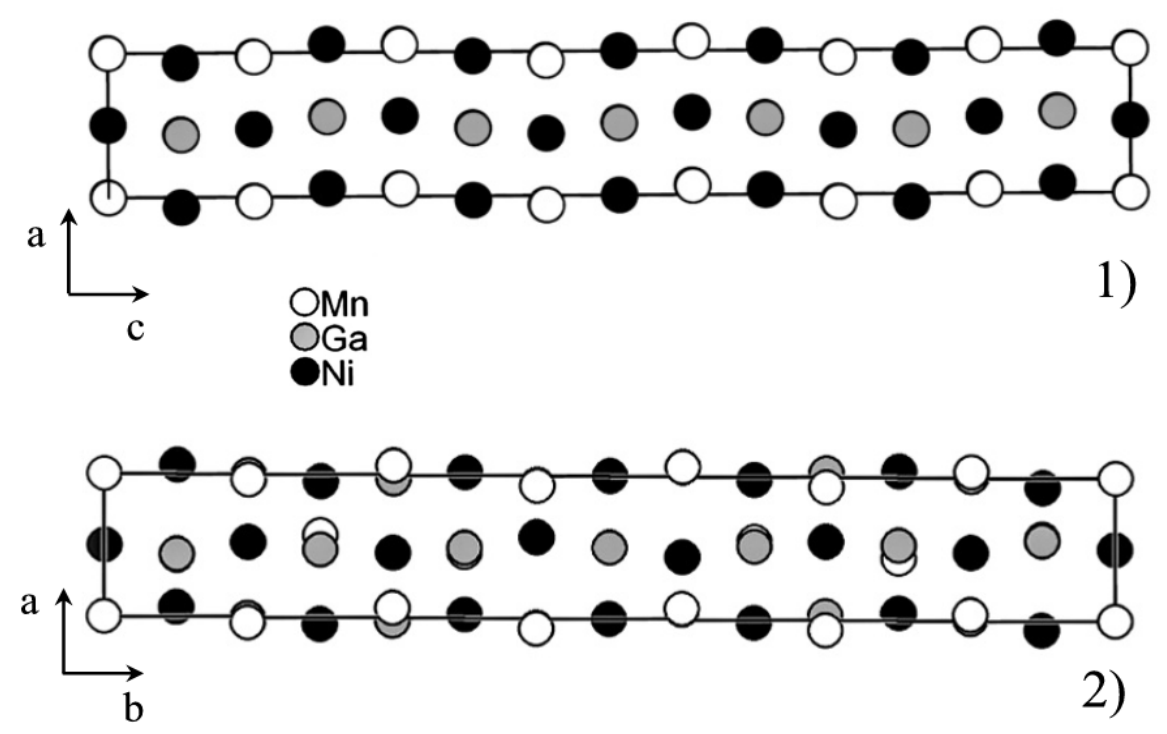

Fig. 3: Comparison of the two seven-fold models for $\mathrm{Ni}_{2} \mathrm{MnGa}$ thermally induced martensite. 1) from Righi et al. ref.[13]. 2) from P.J. Brown ref. [17].

Further structural investigations have demonstrated that the commensurate form of martensitic $5 \mathrm{M}$ modulation occurs in off-stoichiometric Ni-Mn-Ga alloys [14]. In particular a polycrystalline sample of composition $\mathrm{Ni}_{2} \mathrm{Mn}_{1.15} \mathrm{Ga}_{0.85}$ has been studied by $\mathrm{X}$-ray powder diffraction at room temperature. The splitting of some fundamental reflection revealed the presence of a slight monoclinic distortion of the orthorhombic martensitic lattice. The superspace model of $\mathrm{Ni}_{2} \mathrm{MnGa}$ has been therefore adapted to correspond such a monoclinic symmetry. The new superspace group was $\mathrm{I} 2 / \mathrm{m}(\alpha 0 \gamma) 00$ with unvaried atomic positions in the $\mathrm{I} 2 / \mathrm{m}$ basic structure. The Rietveld refinement has been successfully carried out giving final unit cell constants $a_{m}=4.2283(2) \AA$, $\mathrm{b}_{\mathrm{m}}=5.5752(2) \AA, \mathrm{c}_{\mathrm{m}}=4.2003(2) \AA, \beta=90.325(6)$ and $\mathbf{q}=0.3991(2) \mathbf{c} *$. The refined modulation vector $\mathbf{q}$ being coincident to $0.4 \mathbf{c}^{*}=2 / 5 \mathbf{c}^{*}$, demonstrates the commensurateness of the modulation. Therefore the structure of Mn-rich martensite presents a commensurate modulation which can be suitably described by a five-fold superstructure having lattice parameters: $a=a_{m}, b=b_{m}, c=5 * c_{m}$. The modulation function assumed refined parameters very similar to those found for $\mathrm{Ni}_{2} \mathrm{MnGa}$ but the periodicity is actually five-fold commensurate. From the superspace description of the modulated crystal, a classical 3-dimensional structure is straightforwardly obtained (for details see ref [14]). In this case, the advantages provided by the superspace approach appears more evident. In fact the refinement of few parameters ( $A_{1 x}$ and $A_{2 x}$ of Eq. 6) in the (3+1)-dimensional model, instead of nine independent atomic positions for $\mathrm{Ni}, \mathrm{Mn}$ and $\mathrm{Ga}$ in the five-fold supercell, solves the structural modulation. As the homologous incommensurate $5 \mathrm{M}$ structure found for $\mathrm{Ni}_{2} \mathrm{MnGa}$ also this second commensurate form generates 4 equi-spaced satellites in the calculated diffraction pattern. Although $5 \mathrm{M}$ modulation has been always related to a single crystal structure, in some cases Ni-Mn-Ga $5 \mathrm{M}$ martensites were reported to have a monoclinic structure with $\beta$ close to $90.3^{\circ}$ [19] whereas for closely stoichiometric $\mathrm{Ni}_{2} \mathrm{MnGa}$ alloys the basic structure is claimed to assume orthorhombic symmetry [20]. We have demonstrated that for Ni-Mn-Ga Heusler alloys there are at least two different kinds of "5M" modulation: monoclinic commensurate (C) and orthorhombic incommensurate (IC). 
$\mathbf{5 M}$ versus temperature change. For $\mathrm{Ni}_{1.95} \mathrm{Mn}_{1.19} \mathrm{Ga}_{0.86}$ composition the evolution of the unit cell parameters and corresponding modulation vector $\mathbf{q}$ has been studied by increasing the temperature from $80 \mathrm{~K}$ to $273 \mathrm{~K}$ (MT occurs below $300 \mathrm{~K}$ ). The structural changes are monitored by X-ray powder diffraction by using a conventional diffractometer with $\mathrm{CuK} \alpha$ radiation equipped with a low temperature chamber. From $80 \mathrm{~K}$ to $180 \mathrm{~K}$ the martensitic unit cell shows small changes whereas at higher temperatures the structural parameters rapidly move from the starting values. Concerning the basic structure the most interesting result regards the different behaviour of the lattice $a$ and $c$ constants. Whereas the $c$ parameter slightly increases, $a$ shows an opposite behaviour indicating, for this crystallographic direction, a negative linear thermal expansion coefficient. In particular, this lattice parameter changes from $4.240 \AA$ at $190 \mathrm{~K}$ to $4.225 \AA$ at $273 \mathrm{~K}$ with a contraction of about $0.4 \%$. From $180 \mathrm{~K}$ the linear enlargement of $b$ exhibits a different slope indicating an higher sensitivity to temperature.
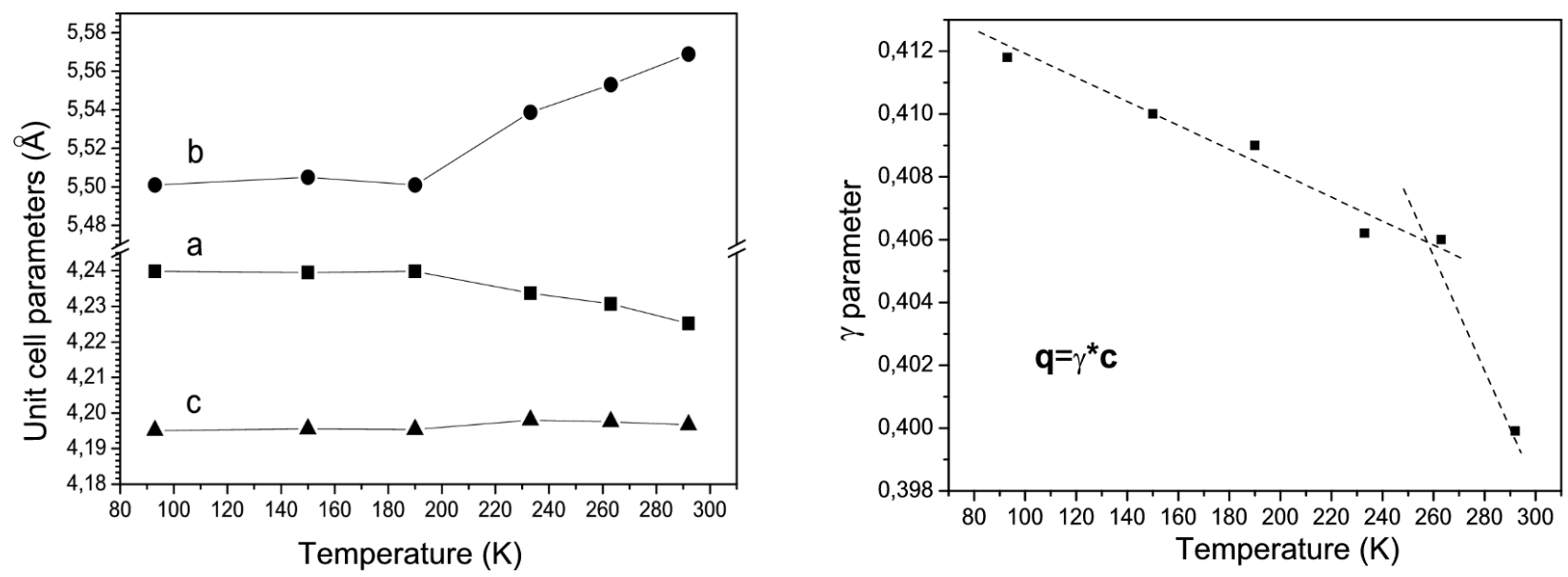

Fig. 4: Left: Graphic of the basic unit cell parameters changes with temperature for commensurate $5 \mathrm{M}(\mathrm{C})$ martensitic phase. Right: corresponding thermal evolution of the modulation vector $\mathbf{q}$. The dashed lines are simply a guide for the eye.

The second plot of Fig. 4 illustrates the temperature dependence of $\mathbf{q}$ modulation vector. At low temperature the $5 \mathrm{M}$ modulation for $\mathrm{NiMnGa}$ is incommensurate being $\gamma$ different from 0.4 . As the temperature increases the periodicity of the modulation continuously varies to reach the five-fold length at $273 \mathrm{~K}$. The $\mathbf{q}$ vector changes with an almost linear behaviour, but, as the room temperature is approached, this variation becomes faster. Therefore when temperature ranges from $83 \mathrm{~K}$ to $200 \mathrm{~K}$, the basic structure is characterized by small changes, whilst the structural modulation remarkably evolves from incommensurate to commensurate. This result clearly indicates that one of the most important parameters, besides the alloy composition, in defining the type of modulation (IC or C) for NiMnGa martensitic phases is the temperature condition.

\section{The "7M" family}

The NiMnGa 7M martensitic structure is commonly associated to the appearing of six satellites between the main reflections of distorted basic structure [7,9,21]. The martensitic basic lattice is usually described as an orthorhombic distortion of the original parent $\mathrm{L} 2{ }_{1}$ structure with $\mathrm{a}>\mathrm{b}>\mathrm{c}$ $[7,12,21]$. However the related I-centred martensitic lattice (see Fig. 1) possesses a strong monoclinic distortion with $\beta \approx 93^{\circ}$. This type of structure has been reported in an experiment where a stoichiometric $\mathrm{Ni}_{2} \mathrm{MnGa}$ single crystal was subjected to external tensile or compression loads along the cubic $\langle 100\rangle$ or $\langle 110\rangle$ crystallographic directions respectively, at room temperature [7]. Furthermore, several investigations evidenced the occurrence of temperature-induced $7 \mathrm{M}$ modulated martensite in Mn-rich [22,23] and Ni-rich [10] Ni-Mn-Ga alloys. Such a Ni-Mn-Ga 
martensite displays some analogies with the Ni-Al layered martensitic structure [14]. The Ni-rich binary $\mathrm{Ni}-\mathrm{Al}$ alloys shows a $7 \mathrm{M}$ martensitic phase [9], with a monoclinic structure having a sevenlayered modulation. This structure has been described in terms of a $(5, \overline{2})$ stacking sequence (related to Zhdanov's notation) of the $(110)_{\mathrm{A}}$ nearly close-packed atomic planes. The same model has been applied to Ni-rich martensitic phase on the basis of TEM analysis [5]. However this kind of studies supply a qualitative description of the structural modulation and our goal was the crystallographic description of the 7M modulated structure. Following the same procedure established for $5 \mathrm{M}$ family we studied off-stoichiometric NiMnGa samples by powder X-ray and neutron diffraction [15].

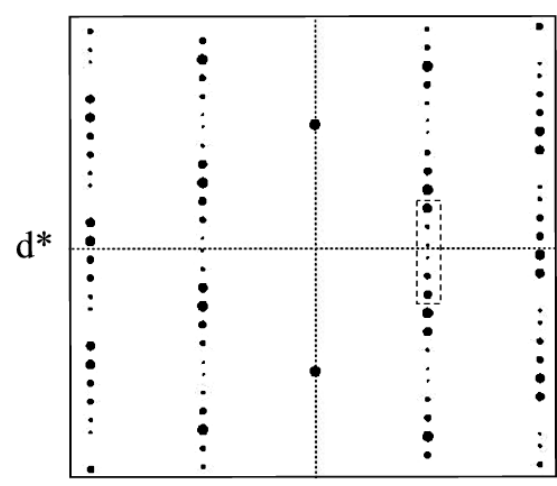

$\mathrm{d}^{*}$

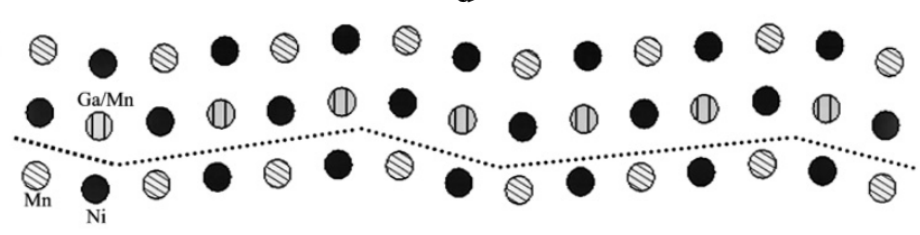

Fig. 5: Bottom: A segment of the 10-fold superstructure related to the incommensurate 7M structure showing the zig-zag distortion of the atomic layers perpendicular to $\mathbf{c}$ axis. Top: corresponding diffraction pattern along [012] fundamental projection. The dashed box evidences the six satellites located between main reflections.

In particular the sample with composition $\mathrm{Ni}_{2} \mathrm{Mn}_{1.2} \mathrm{Ga}_{0.8}$ is found to have a monoclinic unit cell $\mathrm{a}=4.222 \AA \mathrm{b}=5.507 \AA \mathrm{c}=4.267 \AA$ with $\beta=93.3^{\circ}$ at room temperature. The refined modulation vector, $\mathbf{q}=0.308 \mathbf{c}^{*}$, reveals that the structural modulation is not commensurate to a seven-fold layered structure being $\gamma$ variable (see Eq.2) significantly different from $2 / 7$. The monoclinic $(3+1)-$ dimensional model chosen for the Rietveld refinement of the powder diffraction pattern is the same recognized for the " $5 \mathrm{M}$ " commensurate structure [14]. The modelling of the periodic displacive distortion, involving the $(00 l)_{\mathrm{M}}$ atomic layers along the $\mathbf{c}$ fundamental axis, requires the introduction of first and second order of Fourier series of Eq.6. The resulting modulation parameters expressed in Table 1 define a modulation function rather similar to a smoothed zig-zag chain than to a perfect sinusoidal modulation as found for " $5 \mathrm{M}$ " structures. If the modulation vector $\mathbf{q}$ is regarded as approximately equal to $3 / 10 \mathbf{c}^{*}$, the modulated structure of $\mathrm{Ni}_{2} \mathrm{Mn}_{1.2} \mathrm{Ga}_{0.8}$ can be referred to a 10 -fold superstructure with monoclinic $\mathrm{P} 2 / \mathrm{m}$ symmetry and unit cell dimensions: $a=a_{M}, b=b_{M} c=10 c_{M}$ with $\beta=\beta_{\mathrm{M}}$. A segment of this 3-dimensional model is displayed in Fig. 5. The reciprocal space, calculated from the 10 -fold superstructure, reflects the typical peak sequence associated to the $7 \mathrm{M}$ modulation (see top of Fig.5).

Interestingly, a similar incommensurate $7 \mathrm{M}$ modulated structure with almost unvaried $\mathbf{q}$ vector and modulation function has been found for $\mathrm{Ni}_{2.15} \mathrm{Mn}_{0.85} \mathrm{Ga}$ composition at room temperature [15] The 7M modulation appears therefore, independently from the Ni-Mn-Ga composition, always featured by an incommensurate periodicity not reproduced by a seven-fold superstructure. 
This monoclinic $(3+1)$-dimensional structure has been also exploited to solve the martensitic crystal structure of $\mathrm{Ni}_{2.4} \mathrm{Fe}_{0.6} \mathrm{Ga}$ alloy [25]. Although this monoclinic modulated structure, refined on the basis of powder neutron diffraction data, shows all the main structural characteristics exhibited by $7 \mathrm{M}$ modulation the most interesting difference regards the periodicity of the modulation. The incommensurate $\mathrm{Ni}_{2.4} \mathrm{Fe}_{0.6} \mathrm{Ga}$ martensitic phase has a modulation vector with $\mathbf{q} \approx 0.35 * \mathbf{c}$. Such a periodic length cannot be related to a ten-fold or seven-fold superstructure but, on the contrary, it is necessary to represent a large sequence of (001) atomic layers involving 17 adjacent unit cells. The unexpected result, concerning this long superstructure, is the occurrence of 5 and not 6 satellites in the calculated diffraction pattern. Previous TEM experiments performed on $\mathrm{Ni}-\mathrm{Fe}-\mathrm{Ga}$ modulated structures revealed the presence of a new phase indicated as $6 \mathrm{M}$ for the presence of 5 satellites [10]. Therefore the investigated polycrystalline sample could be identified as incommensurate $6 \mathrm{M}$ modulated.

Table 1: Summary of the structural modulation parameters found for different martensitic phases of Heusler FSMA The An(x) coefficients are related to Eq.6.

\begin{tabular}{|l|l|c|c|c|c|}
\hline \multicolumn{1}{|c|}{ Alloy composition } & \multicolumn{1}{|c|}{ Symmetry } & type & $\mathbf{A 1 ( x )}$ & $\mathbf{A 2 ( x )}$ & $\boldsymbol{\gamma}$ \\
\hline $\mathrm{Ni}_{2} \mathrm{MnGa}$ & Orthorhombic & $5 \mathrm{M}(\mathrm{IC})$ & $0.0700(2)$ & -- & $0.4248(2)$ \\
\hline $\mathrm{Ni}_{1.95} \mathrm{Mn}_{1.19} \mathrm{Ga}_{0.86}$ & Monoclinic & $5 \mathrm{M}(\mathrm{C})$ & $0.0677(8)$ & $-0.002(3)$ & $0.3991(2)$ \\
\hline $\mathrm{Ni}_{2} \mathrm{Mn}_{1.2} \mathrm{Ga}_{0.8}$ & Monoclinic & $7 \mathrm{M}(\mathrm{IC})$ & $0.090(1)$ & $0.033(1)$ & $0.30814(4)$ \\
\hline $\mathrm{Ni}_{2.15} \mathrm{Mn}_{0.85} \mathrm{Ga}$ & Monoclinic & $7 \mathrm{M}(\mathrm{IC})$ & $0.069(1)$ & $0.048(2)$ & $0.307(4)$ \\
\hline $\mathrm{Ni}_{2.4} \mathrm{Fe}_{0.6} \mathrm{Ga}$ & Monoclinic & $6 \mathrm{M}(\mathrm{IC})$ & $0.088(1)$ & $0.043(2)$ & $0.3513(5)$ \\
\hline
\end{tabular}

\section{Concluding Remarks}

The present work summarizes a series of structural analysis of different modulated Ni-Mn-Ga martensitic phases of ferromagnetic shape memory Heusler alloys. We found that the structural modulation is frequently incommensurate and in some cases commensurate. The occurrence of incommensurate modulation automatically requires the reconsideration of the structural description based on $n$-fold superstructures. In particular, it has been demonstrated that the correlation between observed satellites and corresponding periodicity of the commensurate models is not valid. The stoichiometric $\mathrm{Ni}_{2} \mathrm{MnGa}$ martensite, related to a seven-fold structure generating a sequence of 4

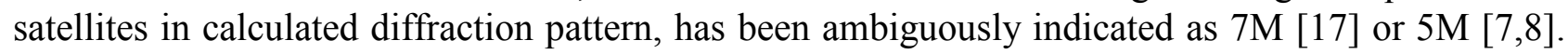
On the contrary, the most important element in determining the possible commensurate model is the modulation vector length. This is also confirmed by the evolution with temperature of $5 \mathrm{M}$ commensurate structure. The martensitic structure departs from the five-fold periodicity assuming incommensurate modulation. The systematic study of different modulated martensitic phases of FSMA provides a new modelling, through superspace, able to successfully describe every " $n \mathrm{M}$ " type of modulation.

\section{Acknowledgments}

The authors are grateful to Dr. Clemens Ritter for his collaboration during neutron powder diffraction experiments performed at Laue-Langevin Institute (ILL), Grenoble, France. It is specially acknowledged the fundamental contribution to the scientific discussion provided by Prof. V.A. Chernenko. 


\section{References}

[1] Advances in Shape Memory Materials. Edited by V.A. Chernenko Vol 583 Materials Science Forum, Trans Tech Pubblications Inc., Switzerland, (2008)

[2] L. Pareti, M. Solzi, F. Albertini, A. Paoluzi: Eur. Phys. J. B Vol. 32 (2003), p. 303

[3] R. C. O’Handley: J. Appl. Phys. Vol. 83 (1998), p. 3263

[4] F. Albertini, L. Morellon, P. A. Algarabel, M. R. Ibarra, L. Pareti, Z. Arnold, and G. Calestani: J. Appl. Phys. Vol. 89 (2001), p. 5614

[5] F. Albertini, L. Pareti, A. Paoluzi, L. Morellon, P.A. Algarabel, M.R. Ibarra, and L. Righi: Appl. Phys. Lett. Vol. 81 (2002), p. 4032

[6] P.J. Webster, K.R.A. Ziebeck, S.L. Town, M.S. Peak: Phil. Mag. Vol. B 49 (1984), p.295

[7] V.V. Martynov, V.V. Kokorin : Journ. de Phys. III Vol. 2 (1992), p.739

[8] V.V. Martynov : Journ. de Phys. IV Vol. C8 (1995), p.91

[9] J.Pons, V.A. Chernenko, R.Santamarta, E.Cesari: Acta Mater. Vol. 48 (2000), p.3027

[10] J. Pons , R. Santamarta, V.A. Chernenko, E. Cesari: Mater. Sci. Eng. A Vol. 438-440 (2006), p.931.

[11] A. Sozinov, A. Likhachev, K. Ullakko: IEEE Trans. Magn. Vol. 38 (2002), p.2814.

[12] P. Mullner, K Ullakko: Phys Stat Sol Vol. B202 (1998), p.R1

[13] L. Righi, F. Albertini, G. Calestani, L. Pareti, A. Paoluzi, C. Ritter, P.A. Algarabel, L. Morellon, M.R. Ibarra: J. Solid State Chem. Vol. 179 (2006), p.3525.

[14] L. Righi L, F. Albertini F, L. Pareti L, A. Paoluzi, G. Calestani: Acta Mater. Vol. 55 (2007), p.5237.

[15] L. Righi, F. Albertini, E. Villa, A. Paoluzi, G. Calestani, V. Chernenko, S. Besseghini, C. Ritter, F. Passaretti: Acta Mater. Vol. 56 (2008), p. 4529.

[16] T. Janssen, A. Janner, A. Looijenga and P.M. Wolff: in International Tables for Crystallography Vol. C, Sect. 9.8., Kluwer, Dordrecht, , (1992)

[17] P.J. Brown, P.J. Crangle, J. Kanomata, T.M. Matsumoto, K.U. Neumann, B. Ouladdiaf, and K. R. A. Ziebeck: J. Phys. Cond. Matter Vol.14 (2002), p.10159.

[18] A.Zheludev, S.M. Shapiro, P. Wochner, L.E. Tanner: Phys. Rev B Vol. 54 (1996), p.15045.

[19] Y. Ge, O. Söderberg, N. Lanska, A. Sozinov, K. Ullakko, V.K. Lindroos: J. Phys IV France Vol.112 (2003), p.112.

[20]Y Noda, S.M. Shapiro, G. Shirane,Y. Yamada, L.E. Tanner: Phys Rev B Vol.42 (1990), p.10397.

[21] A. Sozinov, A.A. Likhachev, N. Lanska, K. Ullakko: Appl. Phys. Lett. Vol.80 (2002), p.1746

[22] U Gaizsch, M. Potschke, S. Roth, N. Mattern, B. Rellinghaus, L. Schultz: J Alloys Comp Vol. 443 (2007), p.99

[23] C. Jiang, Y. Muhammad, L. Deng, W. Wu, H. Xu : Acta Mater Vol. 52 (2004), p.2779.

[24] S. Morito, K. Otsuka: Mater Sci Engign A Vol.208 (1996), p.47

[25] L. Righi, P. Lázpita, J. Gutierrez, J.M. Barandiaran, V.A. Chernenko, G. Calestani : submitted to Scripta Mater. (2009) 